Session 2: Death throes: supernovae, stellar deaths and progenitors 


\title{
The Core-Collapse Supernova Explosion Mechanism
}

\author{
Bernhard Müller ${ }^{1,2}$ \\ ${ }^{1}$ Astrophysics Research Centre, Queen's University Belfast \\ BT7 1NN, Belfast, Northern Ireland \\ email: b.mueller@qub.ac.uk \\ ${ }^{2}$ Monash Centre for Astrophysics, Monash University \\ Clayton, VIC 3800, Australia
}

\begin{abstract}
The explosion mechanism of core-collapse supernovae is a long-standing problem in stellar astrophysics. We briefly outline the main contenders for a solution and review recent efforts to model core-collapse supernova explosions by means of multi-dimensional simulations. Focusing on the neutrino-driven mechanism, we summarize currents efforts to predict supernova explosion and remnant properties.
\end{abstract}

Keywords. Supernovae: general, stars: evolution, stars: interiors, hydrodynamics, instabilities, convection, neutrinos, turbulence, methods: numerical

\section{Introduction}

Many open questions still surround the terminal gravitational core collapse of massive stars. Theory has by now established a clear picture of the first two acts of their death throes. The collapse of the iron (or in some cases $\mathrm{O}-\mathrm{Ne}-\mathrm{Mg}$ ) core is triggered by deleptonization and/or photo-disintegration of heavy nuclei. The collapse is then halted once the core reaches supranuclear densities, and its elastic rebound launches a shock wave that quickly stalls due to energy losses by nuclear dissociation and neutrino losses. Theory has yet to fully explain the subsequent acts in the drama - the revival of the shock and the development of a supernova explosion - let alone the full systematics of supernova explosion and remnant properties. Among the proposed explosion scenarios (see Janka 2012 for an overview), the neutrino-driven mechanism and various flavours of a magnetorotational mechanism have been most thoroughly explored.

Here we shall mostly focus on the neutrino-driven mechanism, which has the virtue of not requiring special evolutionary channels for producing progenitors that spin more rapidly than generically predicted by current stellar evolution models (Heger et al. 2005; Cantiello et al. 2014) for the bulk of massive stars. In the neutrino-driven scenario (sketch in Figure 1), shock revival is accomplished thanks to the increase of the post-shock pressure due to partial reabsorption of neutrinos that stream out from the young protoneutron star (PNS) and the cooling layer of accreted material on its surface. Except for the low-mass end of the progenitor spectrum (e.g. Kitaura et al. 2006; Melson et al. 2015b), this mechanism has been found to depend critically on multi-dimensional (multiD) instabilities such as buoyancy-driven convection (Herant et al. 1994) and the standing accretion shock instability (SASI, Blondin et al. 2003) to foster neutrino-driven runaway shock expansion by a combination of effects including the mixing of the post-shock region and the provision of turbulent stresses (Murphy et al. 2013). Demonstrating quantitatively that shock revival can be achieved in this manner is a notoriously hard problem and requires sophisticated multi-physics simulations that incorporate neutrino transport, 


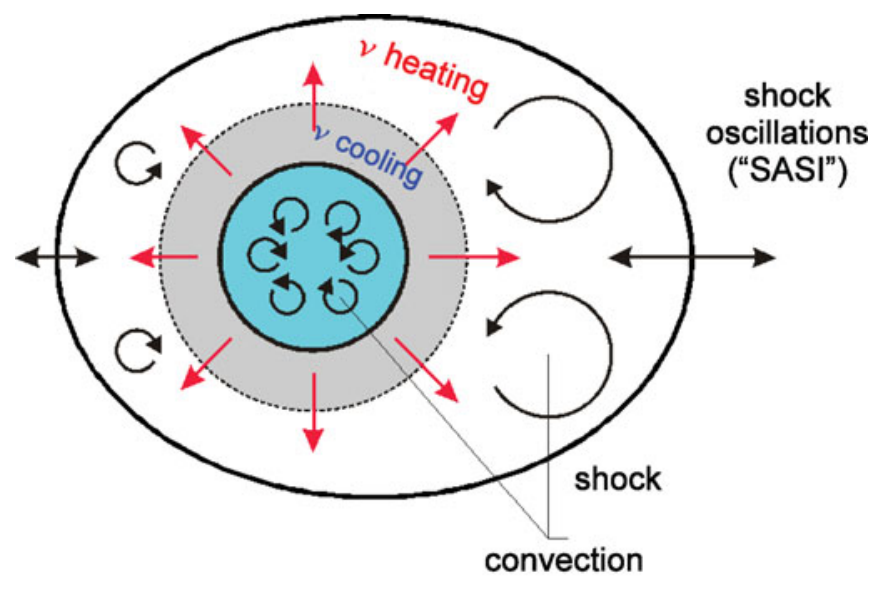

Figure 1. Sketch of the crucial elements of the neutrino-driven explosion mechanism. Neutrinos are emitted from the accretion layer (grey) around the PNS and from its core. A fraction of the electron-flavour neutrinos are reabsorbed in the "gain layer" behind the shock. In the gain region, non-spherical flow can develop because neutrino heating drives convective overturn and because of an advective-acoustic instability (SASI) that manifests itself in the form of large-scale oscillatory motions of the shock. Energy and lepton number loss also drives convection inside the PNS (cyan), which does not play a prominent role for shock revival, however.

multi-D fluid flow, general relativity, and the pertinent microphysics (neutrino interaction rates, nuclear equation of state) at an appropriate level of accuracy.

\section{Progress and Challenges in Modelling Neutrino-Driven Explosions}

As they progressed in sophistication over several decades, simulations of neutrinodriven explosion followed a tortuous path between success and failure. The most recent step to three-dimensional (3D) multi-group neutrino hydrodynamics simulations has been no exception. After experiments with simple "light-bulb" models in 3D (Nordhaus et al. 2010; Hanke et al. 2012; Couch 2013), fully-fledged 3D three -flavour neutrino hydrodynamics simulations soon became available (Hanke et al. 2013), and by now there is already a modest number of successful explosion models for progenitors from $9.6 M_{\odot}$ to $20 M_{\odot}$ obtained with rigorous (Melson et al. 2015b,a; Lentz et al. 2015) or simplified (Takiwaki et al. 2014; Müller 2015) energy-dependent neutrino transport. By and large, the 3D models have exhibited a trend towards missing (Hanke et al. 2012; Tamborra et al. 2014) or delayed explosions, i.e. they show less optimistic conditions for shock revival compared to the massive corpus of successful axisymmetric (2D) simulations that has been amassed by different groups (Janka et al. 2012; Bruenn et al. 2013; Nakamura et al. 2014; Summa et al. 2016; O'Connor \& Couch 2015). The adverse effect of the third dimension has mainly been attributed to the different behaviour of turbulence in 3D and $2 \mathrm{D}$ (though this is only the most important element of a more nuanced view as pointed out by Müller 2016 and Janka et al. 2016). In 2D the inverse turbulent cascade proves conducive to the emergence of large-scale modes and allows convection and the SASI to become more vigorous (Hanke et al. 2012). The heating conditions are not substantially more pessimistic in $3 \mathrm{D}$, however. A quantitative analysis reveals that even non-exploding models, such as the $27 M_{\odot}$ (Hanke et al. 2012), 11.2M $M_{\odot}$, and $20 M_{\odot}$ (Tamborra et al. 2014) runs of the MPA group, come close to the critical conditions for neutrino-driven runaway shock expansion. 
Nonetheless, the results of recent simulations pose a problem. On the one hand, 3D models have not even been able to clearly establish the efficacy of the neutrino-driven mechanism for progenitors in the mass range of $10-15 M_{\odot}$, where there is clear observational evidence for "explodability" (Smartt 2015). This may merely be a result of poor sampling as only one case in the middle of this range has been simulated with state-ofthe-art transport so far (Tamborra et al. 2014). On a more serious note, the delayed onset of explosions in 3D models makes it more difficult to reach significant explosion energies, as the decline of the neutrino luminosities and the mass in the gain region reduces the amount of energy that can be pumped into the ejecta by neutrino heating.

The more reluctant development of explosions in 3D has therefore prompted efforts to identify missing physical ingredients for earlier and more robust shock revival. The gist behind these ideas can be understood if the problem of shock revival is phrased in terms of a critical limiting luminosity $L_{\nu \text {,crit }}$ as a function of accretion rate $\dot{M}$ for stationary accretion flow in spherical symmetry (Burrows \& Goshy 1993). This concept has been generalized to account for other parameters of the accretion flow and multi-D effects (Janka 2012; Müller \& Janka 2015; Janka et al. 2016) in a phenomenological manner and captures the transition to shock revival remarkably well even in multi-D (Summa et al. 2016). In terms of the averaged root-mean-square energy $E_{\nu}$ of $\nu_{e}$ and $\bar{\nu}_{e}$, the accretion rate $\dot{M}$, the PNS mass $M$, the gain radius $R_{\mathrm{g}}$, the average net binding energy $\left|e_{\mathrm{tot}, \mathrm{g}}\right|$ in the gain region, and the average $\left\langle\mathrm{Ma}^{2}\right\rangle$ of the (squared) turbulent Mach number in the gain region, Summa et al. (2016) find

$$
\left(L_{\nu} E_{\nu}^{2}\right)_{\mathrm{crit}} \propto(M \dot{M})^{3 / 5}\left|e_{\mathrm{tot}, \mathrm{g}}\right|^{3 / 5} R_{\mathrm{g}}^{-2 / 5}\left(1+\frac{4}{3}\left\langle\mathrm{Ma}^{2}\right\rangle\right)^{-3 / 5} .
$$

The key to more robust explosions consists in identifying effects that change the terms in Equation (2.1) in a favourable direction.

\subsection{Uncertainties in the Microphysics}

The potential impact of uncertainties in the microphysics on the supernova explosion mechanism has long been recognized. We cannot cover the full spectrum of ideas, which even includes less defensible scenarios for achieving shock revival in spherical symmetry (Fischer et al. 2011). Instead we merely highlight some developments that illustrate that modest (and therefore credible) variations of the input physics could tip the balance in favour of shock revival in multi-D models that are already close to the explosion threshold by increasing $L_{\nu}$ and $E_{\nu}$. This can be achieved, e.g., by nuclear equations of state that are "softer" (Janka 2012; Suwa et al. 2013) in the sense that they result in a faster contraction of the warm PNS and hence in hotter neutrinospheres. Contrary to superficial expectations, the increase of the neutrino luminosities and mean energies outweighs the adverse effect (increase of $\left|e_{\text {tot,g }}\right|$ ) of the contraction of the gain radius.

A similar beneficial effect on the heating conditions could result from faster contraction of the PNS because of enhanced heavy flavour neutrino losses. This was recently explored in 3D simulations by Melson et al. (2015a), who found that a reduction of neutral-current scattering opacities of the order of $\sim 20 \%$ in the neutrinospheric region was sufficient to tip the scale in favour of an explosion in a $20 M_{\odot}$ progenitor. Melson et al. (2015a) achieved this opacity reduction by assuming a stronger strangeness contribution $g_{\mathrm{a}}^{\mathrm{s}}=-0.2$ to the axial vector coupling of the nucleon than measured in current experiments. This is merely a parameterization of nuclear physics uncertainties that can lead to similar effects such as nucleon correlations; here the accuracy of extant rate calculations (Burrows \& Sawyer 1998; Reddy et al. 1999) based on the random phase approximation (RPA) in the relevant density regime may be questioned. More reliable calculations of 


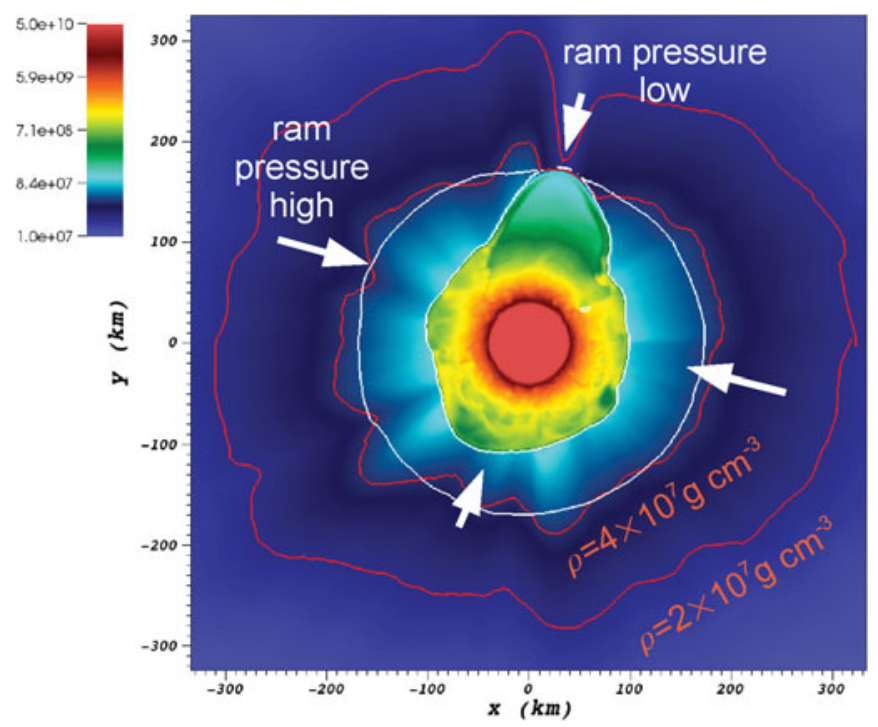

Figure 2. Illustration of the perturbation-aided version of the neutrino-driven mechanism, showing a $2 \mathrm{D}$ slice of the density in a simulation of an $18 M_{\odot}$ star using $3 \mathrm{D}$ initial conditions from Müller (2016) at a time of $293 \mathrm{~ms}$ after bounce. The convective velocity perturbations in the oxygen shell are translated into density perturbations during the collapse as can be seen by the distortion of density isocontours (red), whereas variations in the pre-shock radial velocity $v_{r}$ are small (outer white curve: isocontour for $v_{r}=-4.5 \times 10^{9} \mathrm{~cm} \mathrm{~s}^{-1}$. The variations in ram pressure significantly distort the shock (inner white curve) and facilitate runaway shock expansion by creating large and stable buoyant bubbles.

neutral-current scattering opacities at neutrinospheric densities due to correlation effects are now emerging (Horowitz et al. 2016) and being tested in supernova simulations (Burrows et al. 2016), but whether there are sizable changes to extant models based on RPA opacities remains to be seen.

\subsection{Perturbation-Aided Explosions and Uncertainties in the Progenitor Structure}

Another idea for more robust neutrino-driven explosion models targets the term $\left\langle\mathrm{Ma}^{2}\right\rangle$ in Equation (2.1) and seeks to identify effects that increase the violence of convection and the SASI in order to reduce the critical luminosity. This requires some additional forcing for the instabilities in the gain region, which could arise naturally due to the infall of shells with sizable seed asymmetries from the late convective burning stages (Couch \& Ott 2013; Müller \& Janka 2015). During the collapse, the initial velocity perturbations in such shells give rise to density and pressure perturbations by advectiveacoustic coupling (Lai \& Goldreich 2000). As illustrated in Figure 2, the anisotropic pre-shock ram pressure then leads to increased shock deformation, the development of fast lateral flows downstream of the shock as a result of shock obliquity (Müller \& Janka 2015), and post-shock density perturbations that are translated into turbulent kinetic energy by buoyancy (Müller et al. 2016b).

Various studies employing parameterised initial perturbations (Couch \& Ott 2013, 2015; Müller \& Janka 2015; Burrows et al. 2016) have been conducted and established the conditions under which this "perturbation-aided" mechanism can be effective. Initial convective Mach numbers $\gtrsim 0.05$ and large-scale flow with dominant wavenumbers $\ell \lesssim 4$ are required for a significant reduction of the critical luminosity (Müller \& Janka 2015). More definitive statements require supernova simulations starting from more 
self-consistent 3D initial models. First attempts to evolve massive stars through the last few minutes of convective shell burning before collapse in 3D have been made by Couch et al. (2015) for silicon burning in a $15 M_{\odot}$ progenitor (assuming octant symmetry and a somewhat problematic acceleration of the deleptonization of the iron core) and by Müller et al. (2016b) for oxygen shell burning in an $18 M_{\odot}$ progenitor using a contracting inner boundary condition. Numerical modelling of the final stages of massive stars is still in its infancy, however. Silicon burning in particular presents a major technical obstacle because it needs to be treated with larger nuclear reaction networks than used in current 3D progenitor simulations. Moreover, the 3D progenitors of Couch et al. (2015) and Müller et al. (2016b) have been computed under the proviso that the evolution up to the last minutes before collapse is captured correctly by spherically symmetric stellar evolution models (see below).

The first multi-group neutrino hydrodynamics simulation (Müller 2016) based on the 3D progenitor model of Müller et al. (2016b) showed a strong dynamical effect of the initial perturbations in the oxygen shell. Thanks to the forced shock deformation, a neutrinodriven explosion develops $250 \mathrm{~ms}$ after bounce, whereas shock revival does not take place in a control run with spherically symmetric initial conditions at least for another $300 \mathrm{~ms}$. By contrast, the effect of perturbations was modest in the simpler leakage-based simulations conducted by Couch et al. (2015) using their $15 M_{\odot}$ model. Despite the vastly different simulation methodology employed by these two groups, this may already indicate that large initial perturbations are not a panacea for the neutrino-driven mechanism: 1D stellar evolution models in fact show a huge spread of the parameters determining the efficiency of the perturbation-aided mechanism. The convective Mach numbers in the relevant shells range between $\sim 0.01$ and $\sim 0.15$ at the onset of collapse, and the shell thickness (which determines the dominant angular wavenumber $\ell$ of convective eddies) also varies tremendously. Pre-collapse asphericities may therefore significantly help shock revival in certain progenitors with violent shell burning, but play a subdominant role in the explosion mechanism in others.

While the idea of a perturbation-aided mechanism does not require any fundamental break with 1D stellar evolution models based on mixing length theory (and merely needs an additional initialization step before the onset of collapse), it has also been speculated that the secular evolution of supernova progenitors may be more seriously affected by multi-D processes such as turbulent entrainment at convective boundaries (Meakin \& Arnett 2007), which might change $M$ and $\dot{M}$ in Equation (2.1). At this stage, it still appears premature to draw conclusions based on 3D simulations of convective boundary mixing (Meakin \& Arnett 2007; Müller et al. 2016b; Cristini et al. 2016; Jones et al. 2017) during brief intervals of advanced burning stages. More work is needed to translate the findings from such multi-D models into suitable recipes for $1 \mathrm{D}$ stellar evolution.

\subsection{Strong SASI, Rapid Rotation, and Magnetic Fields}

Several other mechanisms for facilitating neutrino-driven shock revival have also been explored recently, although it is less clear whether they could operate generically. Fernández (2015) found a reduction of the critical luminosity in the strongly SASI-dominated regime in $3 \mathrm{D}$ compared to $2 \mathrm{D}$ in his light-bulb models, which he traced to the ability of the non-axisymmetric SASI spiral mode to store more non-radial kinetic energy than the axisymmetric sloshing mode in the non-linear regime. Whether and when this regime is realized remains to be further investigated; the effect has not yet been replicated by self-consistent simulations that probe the SASI-dominated regime (e.g. the $27 M_{\odot}$ and $20 M_{\odot}$ models of Hanke et al. 2013 and Melson et al. 2015a), and may be restricted to massive progenitors with sustained high accretion rates. 
Janka et al. (2016) and Takiwaki et al. (2016) showed that rapid rotation is conducive to shock revival even without the help of magnetic fields. For increasing rotation rate, this beneficial effect initially comes about because the contribution of rotational energy reduces the average net binding energy $\left|e_{\text {tot,g }}\right|$ in the gain region, and because angular momentum support lowers the pre-shock velocity (Janka et al. 2016). Above a critical rotation rate corresponding to initial iron core spin periods of $\sim 1 \mathrm{~s}$, the effect of rotation becomes more dramatic as a strong corotation instability develops (Takiwaki et al. 2016).

There may also be a regime where magnetic fields play a subsidiary role in the neutrinodriven mechanism instead of acting as the primary driver of the explosion if the initial fields can be sufficiently amplified by a small-scale turbulent dynamo, which could be provided by convection (Thompson \& Duncan 1993) or by the SASI (Endeve et al. 2010, 2012) even in the absence of rapid rotation. If the fields come close to equipartition strength, they may help organize the flow into stable large-scale bubbles and thereby prove conducive to shock revival (Obergaulinger et al. 2014). However, strong initial fields of $\sim 10^{12} \mathrm{G}$ are still required for this scenario in Obergaulinger et al. (2014)

If either rotation or magnetic fields are to play at least a subsidiary role in neutrinodriven explosions, it thus appears that special evolutionary channels are already required considering that current stellar evolution models predict pre-collapse spin periods of $\gtrsim 30 \mathrm{~s}$ and magnetic fields of $\lesssim 10^{10} \mathrm{G}$ (Heger et al. 2005) for solar-metallicity supernova progenitors.

\subsection{The Need for Alternative Mechanisms for Hypernovae}

That extremely fast rotation rates are encountered at least in a small fraction of supernova progenitors is suggested by observations of broad-lined Ic supernovae or "hypernova", whose explosion energies reach up to $\sim 10^{52} \mathrm{erg}$ (Drout et al. 2011). These energies are likely out of reach for the neutrino-driven mechanism and thus require a different explosion mechanism altogether; even optimistic parameterised models suggest an upper limit of $\sim 2 \times 10^{51} \mathrm{erg}$ for neutrino-driven explosions. Various magnetohydrodynamic mechanisms (Usov 1992; MacFadyen \& Woosley 1999; Akiyama et al. 2003) that tap the rotational energy of a rapidly spinning and highly magnetized "millisecond magnetar", black hole, or accretion disk remain the most promising explanations for the most energetic supernovae and the gamma-ray-bursts associated with some of them, but we must refer to the review of Janka (2012) for a more detailed discussion.

\section{Outlook: From Neutrino-Driven Explosion Models to Observables}

It is conceivable that $3 \mathrm{D}$ simulations of core-collapse supernovae may need no more than a combination of relatively minor changes in the input physics and slight improvements in numerical accuracy (for a discussion see Janka et al. 2016 and Müller 2016) to produce neutrino-driven explosions over a wide range of progenitor masses. This, however, would only provide a solution to the problem of shock revival. The bigger challenge lies in accounting for the observed explosion (e.g. explosion energy and nickel mass) and remnant properties (neutron star mass, spin, and kick).

In neutrino-driven supernovae, the key explosion properties only reach their asymptotic values after a phase of simultaneous accretion and mass ejection that lasts for $\gtrsim 1 \mathrm{~s}$ after shock revival (or even later in the case of the neutron star kicks, see Wongwathanarat et al. 2013). Such time-scales are only marginally within reach even for 3D hydrodynamics simulations with simplified multi-group neutrino transport (Müller 2015, 2016). Axisymmetric models do not offer a serious alternative not only because of their higher proclivity to explosion. $2 \mathrm{D}$ effects are even more problematic during the explosion phase 
than prior to shock revival (Müller 2015) and may be responsible for the low explosion energies (compared to typical observed values of $5 \ldots 9 \times 10^{50} \mathrm{erg}$, see Kasen \& Woosley 2009) and high neutron star masses found in typical 2D simulations (Janka et al. 2012; Nakamura et al. 2015; O'Connor \& Couch 2015, but see also the more energetic models of Bruenn et al. 2016). Because of these obstacles, it yet remains to be demonstrated by self-consistent models that the neutrino-driven mechanism can account for the explosion properties of the majority of core-collapse supernovae (and this statement holds a fortiori for the magnetohydrodynamic mechanism).

At present, the only alternative for understanding the systematics of the explosion properties is to rely on parameterised models of neutrino-driven supernovae (e.g. O'Connor \& Ott 2010; Ugliano et al. 2012; Pejcha \& Thompson 2015; Ertl et al. 2016; Sukhbold et al. 2016; Müller et al. 2016a) that are based on 1D simulations and/or analytic theory. Although objections may be raised against the predictiveness of such an approach, it has undoubtedly proved valuable and led to significant results. Although the available parameterised models differ greatly in detail, some findings such as trends in "explodability" (with explosions up to $\sim 15 M_{\odot}$ and several islands of explodability alternating with black hole formation up to $\sim 30 M_{\odot}$ ), or the upper limit of $\sim 2 \times 10^{51} \mathrm{erg}$ (Ugliano et al. 2012; Ertl et al. 2016; Sukhbold et al. 2016; Müller et al. 2016a) have proved quite robust, which suggests that they indeed reflect the inherent physics of the neutrino-driven mechanism. Furthermore, first-principle simulations are increasingly starting to inform parameterised models (Müller et al. 2016a), and these in turn are proving useful for selecting appropriate targets for full-scale multi-D simulations. Given the prohibitive costs of state-of-the-art models, such a two-pronged approach likely remains the best strategy for explaining the diversity of core-collapse supernovae in the foreseeable future.

\section{Acknowledgements}

The author acknowledges long-term assistance by his collaborators, especially A. Heger, H.-Th. Janka, and T. Melson, and support by the STFC DiRAC HPC Facility (DiRAC Data Centric system, ICC Durham), the National Computational Infrastructure (Australia), the Pawsey Supercomputing Centre (University of Western Australia), and the Minnesota Supercomputing Institute.

\section{References}

Akiyama, S., Wheeler, J. C., Meier, D. L., Lichtenstadt, I. Meier, D. L., \& Lichtenstadt. 2003, ApJ, 584, 954

Blondin, J. M., Mezzacappa, A., \& DeMarino, C. 2003, ApJ, 584, 971

Bruenn, S. W. et al. 2016, ApJ, 818, 123

—. 2013, ApJL, 767, L6

Burrows, A. \& Goshy, J. 1993, ApJL, 416, L75+

Burrows, A. \& Sawyer, R. F. 1998, Phys. Rev. C, 58, 554

Burrows, A., Vartanyan, D., Dolence, J. C., Skinner, M. A., \& Radice, D. 2016, ArXiv e-prints, 1611.05859

Cantiello, M., Mankovich, C., Bildsten, L., Christensen-Dalsgaard, J., \& Paxton, B. 2014, ApJ, 788,93

Couch, S. M. 2013, ApJ, 775, 35

Couch, S. M., Chatzopoulos, E., Arnett, W. D., \& Timmes, F. X. 2015, ApJL, 808, L21

Couch, S. M. \& Ott, C. D. 2013, ApJL, 778, L7

- 2015, ApJ, 799, 5

Cristini, A., Meakin, C., Hirschi, R., Arnett, D., Georgy, C., \& Viallet, M. 2016, Phys. Scr., 91, 034006

Drout, M. R. et al. 2011, ApJ, 741, 97 
Endeve, E., Cardall, C. Y., Budiardja, \& Mezzacappa, A. 2010, ApJ, 713, 1219

Endeve, E., Cardall, C. Y., Budiardja, R. D., Beck, S. W., Bejnood, A., Toedte, R. J., Mezzacappa, A., \& Blondin, J. M. 2012, ApJ, 751, 26

Ertl, T., Janka, H.-T., Woosley, S. E., Sukhbold, T., \& Ugliano, M. 2016, ApJ, 818, 124

Fernández, R. 2015, MNRAS, 452, 2071

Fischer, T. et al. 2011, ApJS, 194, 39

Hanke, F., Marek, A., Müller, B., \& Janka, H.-T. 2012, ApJ, 755, 138

Hanke, F., Müller, B., Wongwathanarat, A., Marek, A., \& Janka, H.-T. 2013, ApJ, 770, 66

Heger, A., Woosley, S. E., \& Spruit, H. C. 2005, ApJ, 626, 350

Herant, M., Benz, W., Hix, W. R., Fryer, C. L., \& Colgate, S. A. 1994, ApJ, 435, 339

Horowitz, C. J., Caballero, O. L., Lin, Z., O'Connor, E., \& Schwenk, A. 2016, ArXiv e-prints, 1611.05140

Janka, H.-T. 2012, Annual Review of Nuclear and Particle Science, 62, 407

Janka, H.-T., Hanke, F., Hüdepohl, L., Marek, A., Müller, B., \& Obergaulinger, M. 2012, Progress of Theoretical and Experimental Physics, 2012, 010000

Janka, H.-T., Melson, T., \& Summa, A. 2016, Annual Review of Nuclear and Particle Science, $66,341,1602.05576$

Jones, S., Andrassy, R., Sandalski, S., Davis, A., Woodward, P., \& Herwig, F. 2017, MNRAS, 465,2991

Kasen, D. \& Woosley, S. E. 2009, ApJ, 703, 2205

Kitaura, F. S., Janka, H.-T., \& Hillebrandt, W. 2006, A\& $A, 450,345$

Lai, D. \& Goldreich, P. 2000, ApJ, 535, 402

Lentz, E. J. et al. 2015, ApJL, 807, L31

MacFadyen, A. I. \& Woosley, S. E. 1999, ApJ, 524, 262

Meakin, C. A. \& Arnett, D. 2007, ApJ, 667, 448

Melson, T., Janka, H.-T., Bollig, R., Hanke, F., Marek, A., \& Müller, B. 2015a, ApJL, 808, L42

Melson, T., Janka, H.-T., \& Marek, A. 2015b, ApJL, 801, L24

Müller, B. 2015, MNRAS, 453, 287 2016, PASA, 33, e048

Müller, B., Heger, A., Liptai, D., \& Cameron, J. B. 2016a, MNRAS, 460, 742

Müller, B. \& Janka, H.-T. 2015, MNRAS, 448, 2141

Müller, B., Viallet, M., Heger, A., \& Janka, H.-T. 2016b, ApJ, 833, 124

Murphy, J. W., Dolence, J. C., \& Burrows, A. 2013, ApJ, 771, 52

Nakamura, K., Kuroda, T., Takiwaki, T., \& Kotake, K. 2014, ApJ, 793, 45

Nakamura, K., Takiwaki, T., Kuroda, T., \& Kotake, K. 2015, PASJ, 67, 107

Nordhaus, J., Burrows, A., Almgren, A., \& Bell, J. 2010, ApJ, 720, 694

Obergaulinger, M., Janka, H.-T., \& Aloy, M. A. 2014, MNRAS, 445, 3169

O'Connor, E. \& Couch, S. 2015, ArXiv e-prints, 1511.07443

O'Connor, E. \& Ott, C. D. 2010, Classical and Quantum Gravity, 27, 114103

Pejcha, O. \& Thompson, T. A. 2015, ApJ, 801, 90

Reddy, S., Prakash, M., Lattimer, J. M., \& Pons, J. A. 1999, Phys. Rev. C, 59, 2888

Smartt, S. J. 2015, PASA, 32, 16

Sukhbold, T., Ertl, T., Woosley, S. E., Brown, J. M., \& Janka, H.-T. 2016, ApJ, 821, 38

Summa, A., Hanke, F., Janka, H.-T., Melson, T., Marek, A., \& Müller, B. 2016, ApJ, 825, 6, 1511.07871

Suwa, Y., Takiwaki, T., Kotake, K., Fischer, T., Liebendörfer, M., \& Sato, K. 2013, ApJ, 764, 99

Takiwaki, T., Kotake, K., \& Suwa, Y. 2014, ApJ, 786, 83

—. 2016, MNRAS, 461, L112

Tamborra, I., Hanke, F., Janka, H.-T., Müller, B., Raffelt, G. G., \& Marek, A. 2014, ApJ, 792, 96

Thompson, C. \& Duncan, R. C. 1993, ApJ, 408, 194

Ugliano, M., Janka, H.-T., Marek, A., \& Arcones, A. 2012, ApJ, 757, 69

Usov, V. V. 1992, Nature, 357, 472

Wongwathanarat, A., Janka, H.-T., \& Müller, E. 2013, A\& A, 552, A126 